\title{
A 3-Dimensional Database of Deep Brain Functional Anatomy, and Its Application to Image-Guided Neurosurgery
}

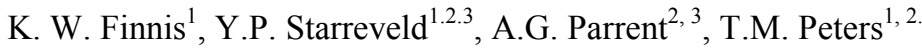 \\ Robarts Research Institute $^{1}$ and Department of Medical Biophysics ${ }^{2}$, \\ Division of Neurosurgery, London Health Sciences Centre ${ }^{3}$ \\ University of Western Ontario, London, Ontario, Canada
}

\begin{abstract}
We describe a surgical planning environment that permits the determination or refinement of the location of a therapeutic neurosurgical intervention using information derived from an electrophysiological database. Such intraoperative stimulation-response and microelectrode recording data are generated from subcortical exploration performed as part of neurosurgical interventions at multiple centres. We have quantified and nonlinearly registered these intraoperative data, acquired from a large population of patients, to a reference brain imaging volume to create an electrophysiological database. This database can then be nonlinearly registered to future patient imaging volumes, enabling the delineation of surgical targets, cell types, and functional and anatomical borders prior to surgery. The user interface to our system allows the population-acquired physiology information to be accessed in a fully searchable format within a patient imaging volume. This system may be employed in both preoperative planning and intraoperative guidance of stereotactic neurosurgical procedures. We demonstrate preliminary results illustrating the use of this database approach to predict the optimum surgical site for creating thalamic lesions in the surgical treatment of Parkinson's disease.
\end{abstract}

\section{Introduction}

Image-guided neurosurgery (IGNS) systems provide neurosurgeons with image-based information that allow neurosurgical sites to be precisely targeted. Such precision is only possible when the target can be seen on the preoperative images of the patient. When desired targets are functionally but not anatomically distinct, as in functional procedures such as pallidotomy or thalamotomy, an approximate target position can only be determined by linear scaling of standardized coordinates [1] or through the use of anatomical atlases fit to anatomical landmarks identified on preoperative scans $[2,3]$. To refine this initial target into a final target, multiple exploratory trajectories with a recording and/or stimulating electrode are required to characterize the function of the intended target and the tissue surrounding it.

Electrophysiological exploration of the living brain may involve any or all of microelectrode recording, microstimulation, or macrostimulation. Microelectrode 
recording within brain tissue identifies neurons that produce characteristic firing patterns in response to specific stimuli and allow characterization of function within that particular region of anatomy. All distinct cell-firing patterns encountered along a trajectory, along with the brain coordinates from which they were evoked are entered into the surgical $\log$ for later analysis. Micro- and macrostimulation introduce electrical current into a discrete area of brain to produce neuronal membrane depolarization. When stimulation supplies enough current to reach neuronal threshold and excite a small pool of neurons, effects are immediately demonstrated or described by the patient. These stimulation-induced effects may take the form of paresthesias (sensory phenomena), muscle contractions, flashing lights in the visual field, general systemic effects, or alteration of the pathophysiological condition (e.g. cessation of tremor). All verbal responses provided by the patient are entered in the surgical log along with electrode position and the current or voltage settings used to evoke the response.

Many of the surgical targets within the brain, localized through the use of stimulating/recording electrodes, are within anatomical structures that are somatotopically organized. Such structures contain a representation of the human body, which "maps" sub-regions of the deep brain structure to discreet body regions in the same manner that Penfield's homunculus maps the functional organization of the primary somatosensory cortex [4]. Physical responses elicited by the patient, descriptions, and microelectrode recording data obtained during exploration aid the surgeon in mentally reconstructing the somatotopic organization contained within the target structure and in establishing functional borders. Comparison of the patient somatotopic organization with that provided by the literature allows estimation of the location of the probe tip within the target and is used in planning subsequent trajectories.

Each passage of the electrode carries a risk of intracranial hemorrhage. Increasing initial targeting accuracy and decreasing the total number of required trajectories to place a lesion or chronic stimulator is therefore highly advantageous. To accomplish this goal, displaying a map of functional organization with the patient's imaging data set prior to surgery is necessary and has been the subject of several prior endeavors $[5,6,7,8]$.

\section{System Description}

\subsection{Database Construction}

During a typical surgical procedure for Parkinson's disease, a preliminary target is indicated on a patient preoperative image, and intraoperative electrophysiological exploration is performed within that anatomical region with the aid of standard stereotactic instrumentation. The function of the region is characterized either by analysis of neural firing patterns obtained during microelectrode recording or by 
application of electrical stimulation via micro- or macrostimulation electrodes and observing the effect on the patient. Each probe trajectory investigated generates many data elements that must be recorded and interpreted intraoperatively. Historically this has been achieved via dictation or in written format by the surgeon. Our system permits direct entry of these data into the visualization/planning system, although historical data may be entered retrospectively. Prior to inclusion of patient data into a normalized database of function, all electrophysiological data are quantified using a coding structure similar to Tasker's [9], a task which in our system is considerably facilitated through the use of a novel graphical user interface (GUI) as illustrated in Figure 1. The coding

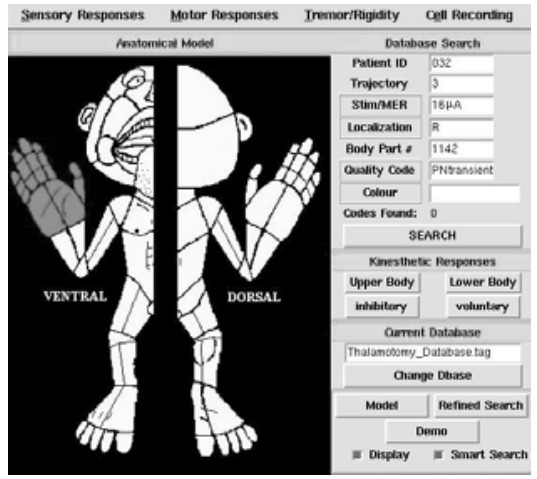

Figure 1. Graphical user interface used to search and enter data into the database. The homunculus figure is employed to define the body part to which the stimulus representation is related. structure incorporates the following data:

1. A patient identification number;

2. A trajectory identification number indicating the trajectory from which data were acquired;

3. The method used for data acquisition (i.e. microelectrode recording, microstimulation, or macrostimulation), and if stimulation was applied, the current or voltage, frequency, and duration of the pulse used to evoke the response;

4. The side or sides of the body which evoked the observed modification of cellular firing or experienced effects upon stimulation;

5. A body part number assigned to that discreet region of the body, and;

6. A modifiable response code describing the quality of the response or changes in cell firing patterns. Typical response codes would indicate the involvement of motor, sensory, or autonomic effects; adverse or positive consequences to the physical condition; cognitive changes, or no effect or cellular response.

Within our image-guided neurosurgery system, the coded functional data are plotted directly onto the patient's preoperative image along a virtual trajectory corresponding to the physical probe position and orientation at the location the data were produced. 
For stereotactic procedures, the data points and trajectory are entered into patient image-space via their coordinates in stereotactic frame space. When tagged to the patient imaging volume, the $\mathrm{x}, \mathrm{y}$, and $\mathrm{z}$ Cartesian coordinates of the functional data in patient image-space relative to the trajectory origin and the corresponding response codes are saved in a text file for addition to the central database. We also assign a secondary code to the header of each data file that describes the sex, age, pathological condition, procedure and handedness of the patient. Any other information considered surgically relevant may be appended to this secondary code. When fully coded and archived, patient data is added to the central database as described below.

\subsection{Non-linear Registration}

We employ a nonlinear registration algorithm "ANIMAL" [10], to nonlinearly register (warp) the patient's preoperative image to a standard, high resolution MRI reference brain, which consists of 27 registered T1-weighted MRI scans of the same individual averaged into a single volume. This data set provides excellent clarity of anatomical structure and a high signal to noise ratio [11]. Upon successful nonlinear registration, the deep brain anatomy of the patient image (source volume) appears morphologically identical to the corresponding anatomical region of the reference brain (target volume). Note that since we are only interested in deep brain structures, we do not require that the patient's cortical sulci and gyri be accurately matched.

For each patient image matched to the reference brain, ANIMAL generates a nonlinear deformation grid that accurately describe a one-to-one mapping of the source to the target volume. This three-dimensional deformation grid is used to re-map the coded patient electrophysiologic data to the target volume. This same nonlinear registration technique may be used to map the reference brain to any arbitrary patient's brain. The deformation matrix generated as part of this procedure can then be employed to map points in the population-based electrophysiology database to the anatomy of one brain without the involvement of standard anatomical atlases or linear scaling techniques.

\section{Surgical Planning}

We have incorporated the electrophysiological atlas described above into our surgical planning environment, so that the atlas information can be used to refine the lesion targets calculated by traditional means (through the use of anatomical atlases or standardized stereotactic coordinates). We believe that it will be possible to use these atlas data as the sole predictor of target loci. Using the inverse of the patient-specific nonlinear transformation matrix generated by nonlinear warping of the patient brain dataset to the reference brain dataset, all data within the functional atlas is transformed into patient image-space as described above. Once transformed, the surgeon may selectively search the database that now conforms to the patient anatomy and obtain electrophysiologic data representative of the desired target. By appropriate selection 
and display of the functional data from the database, probabilistic functional boundaries within the anatomically homogeneous (MRI) anatomy may be displayed, or alternatively, the locations where the intended therapy was most effective in past patients can be highlighted. Extracted database information is displayed within the IGNS system at the appropriate patient coordinates incorporating user-specified parameters, such as colour, shape representation, and opacity.

The electrical stimulation points extracted from the database are automatically scaled in size to model both the amount of current (microstimulation) or voltage (macrostimulation) used to evoke the response and the resulting spread of current through brain tissue. This scaled representation provides the user with the probable spatial extent of the neurons involved when that particular response was evoked by the stimulation parameters specified in the code. This indicates that while a particular response was evoked at that particular coordinate within the patient brain, neurons responsible for producing the response may have existed anywhere within that volume. For a more comprehensive probabilistic analysis, the surgeon may define a virtual sphere of arbitrary radius at any point in the patient dataset and search the database for all code coordinates that lie within the volume of that sphere. The results of such a search are displayed as a breakdown of all responses that lie within the virtual sphere, as a percentage of the total number of responses. All codes found within the sphere that describe kinesthetic responses are further classified into percentage of responses for specific joints and movements around the joint; muscle contractions are subdivided into discreet muscle groups; and sensory phenomena are arranged into type and their location on the body. This virtual search function is fully customizable by the user to suit individual requirements.

\section{Intra-operative Guidance}

Intraoperatively, comparison of responses observed during electrophysiological exploration, with those predicted by the patient-registered database, provides instantaneous validation of database conformity and displays archived electrophysiologic data in a surgically relevant manner. If lesioning is to be performed, the neurosurgeon can determine the size of the therapeutic lesion or combination of lesions that will optimally destroy or deactivate the anticipated target resulting in minimal trauma to surrounding structures. Accordingly, upon validation of its predictive ability, the database may be used as the sole source of surgical target localization and planning (at the discretion of the surgeon) or used in conjunction with pre-existing localization techniques to refine an approximated target.

\section{Initial Clinical Results}

The database currently contains information from 75 patients from two clinical sites. At this point all the electrophysiological data have been entered into the database 
retrospectively, but we are nevertheless in a position to demonstrate the effectiveness of this system to predict the probable location of a lesion to treat Parkinson's disease. Figure 2 demonstrates several clusters of sensory and motor responses positioned in 3$\mathrm{D}$ within patient brain space. a) displays the region of the patient brain (the left thalamus) within which the database points are located; b) shows the location of tremor cells for the population data, and c) demonstrates the trajectory of a lesioning probe that would ablate the region containing the tremor responses. Also shown in c) is the somatotopy exhibited by the thalamic sensory nucleus as predicted by the database. Note that this particular patient's intraoperative data were not contained in the database used in the following demonstration.
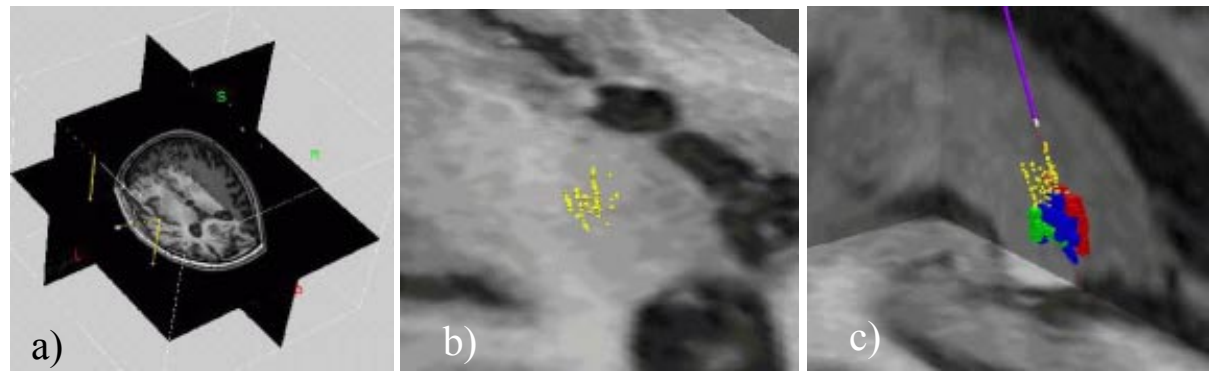

Figure 2: a) Context for database application; b) Axial: Tremor cells within the Ventralis intermedius (kinesthetic) nucleus; c) Sagittal: Functional border between kinesthetic motor nucleus (small spheres) and Ventralis caudalis sensory nucleus (larger cubes). Somatotopy is evident within the Ventralis caudalis (tongue, fingers and foot sensory regions, from right to left). Trajectory of lesioning probe is also shown in c).
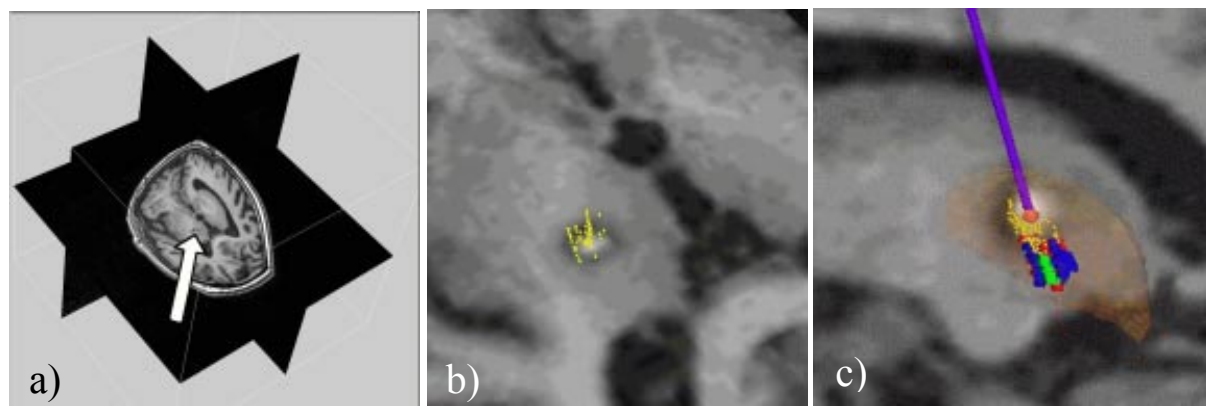

Figure 3: Post-op MRI. a) Context, showing site of applied lesion. Arrow points to lesion; b) Axial: cluster of tremor cells from database, shown with respect to lesion; c) Sagittal: Tremor cells and sensory nucleus data with respect to lesion. Lesioning electrode modeled to reflect orientation and position of the physical probe that produced the lesion.

Figure 3 shows the same database information mapped to the post-operative scan of the same patient following surgery. We note that the surgery of this patient was planned and performed using standard techniques, and did not take advantage of the 
database contents. Nevertheless, it is clearly demonstrated by figure $3 \mathrm{~b}$ that the cluster of tremor cells lies almost completely within the lesion volume. Fig $3 \mathrm{c}$ shows the point clusters as seen in Figure 2, located within the context of the anatomy of the thalamus.

We emphasize that these are preliminary results. Nevertheless, they give us the confidence to proceed to the next stage of this work that will encompass rigorous validation of our registration and targeting strategies, as well as correlating our results with clinical follow-up data.

\section{Discussion}

This method has several advantages over previous work. Nonlinear registration provides the greatest advantage by accommodating for the neuroanatomical variability found between patients when data are added to the reference brain database and subsequently registered to patient images. None of the earlier work in this field incorporates nonlinear registration (warping) techniques. Further, mapping intraoperative data to the patient image with this technique does not require initial tagging of data to representative slices of digitized anatomical atlases or require anatomical landmarks for the determination of scaling factors to fit the data to images of the patient or reference brain. The improvements offered by our technique can be demonstrated by the fact that functional targets are instantaneously delineated on preoperative images as distinct clusters of functionally related data, without the randomness and scatter seen when linear scaling is employed with anatomical atlasbased approaches $[12,13]$.

Clustered population electrophysiologic data will display somatotopic organization within proposed targets (Figures 2,3) and may be selectively searched to find specific physiologic regions within the target. Another improvement of this technique is the level of coding detail incorporated. In the past, coding detail contained was neither sufficient to accurately describe an observed response in relation to the parameters that evoked it nor to enable the user to trace the origin of the code back to the original patient or trajectory from which it was produced. Our coding method and database design not only make this possible but permits the user to retrieve data from specific age groups and sex, data evoked from patients with a particular handedness, or codes obtained from patients with specific diseases. By accessing our database through a flexible, user-friendly interface that facilitates detailed searches for any user-defined parameters, our coding technique is ideally suited for intraoperative guidance, preoperative planning and postoperative analysis of electrophysiological data.

A digital probabilistic atlas that utilizes population data will improve in accuracy over time, achieving better statistics with the addition of more data. While only qualitative results were presented in this paper, with 75 patients currently in the database providing over 6100 data points, we are now in a position to commence validation studies that quantitatively assess its design and contents. 


\section{References}

1. Favre J., Taha J.M. Nguyen T.T., Gildenberg P.L.,Burchiel K.J. Pallidotomy: A survey of current practice in North America. Neurosurgery 39:883-892, 1996

2. St-Jean, P., Sadikot, A. F., Collins, D. L., Clonda, D., Kasrai, R., Evans, A. C., and Peters, T. M., Automated atlas integration and interactive 3-dimensional visualization tools for planning and guidance in functional neurosurgery, IEEE Trans Medical Imaging, vol. 17, no. 5, pp. 672-680, 1998.

3. Yeo T.T., Nowinsky W.L. Functional neurosurgery aided by use of an electronic brain atlas. Acta Neurochir [Suppl] 68:93-99, 1997

4. Penfield W., Rasmussen T. The Cerebral Cortex of Man: A Clinical Study of Localization of Function. Macmillan, New York, 1950.

5. Thompson, C. J., Hardy, T. L., and Bertrand, G. A system for anatomical and functional mapping of the human thalamus. Comput Biomed Res 10, 9-24. 1977

6. Tasker R.R., Hawrylyshyn P., Organ W. Computerized graphic display of physiological data collected during human stereotactic surgery. Appl Neurophysiol 41:193-187, 1978.

7. Yoshida M., Okada K., Nagase A., Kuga S., Watanabe M., Kuramoto S. Neurophysiological atlas of the human thalamus and adjacent structures. Appl Neurophysiol 45:406-409, 1982.

8. Hardy TL. A method for MRI and CT mapping of diencephalic somatotopography. Stereotact Funct Neurosurg 52:242-249, 1989.

9. Tasker R.R., Hawrylyshyn P., Organ W. Computerized graphic display of physiological data collected during human stereotactic surgery. Appl Neurophysiol 41:193-187, 1978.

10. Collins D.L., Peters T.M., and Evans A.C. An automated 3D non-linear image deformation procedure for determination of gross morphometric variability in the human brain. Robb, R. A. 2359, 180-190. 1994. Seattle WA, SPIE. Proceedings of the Third Conference on Visualization in Biomedical Computing.

11. Holmes C.J., Hoge R., Collins D.L., Woods R., Toga A.W., and Evans A.C., Enhancement of MR images using registration for signal averaging. JCAT 22:324333, 1998.

12. Hardy T.L., Bertrand G., Thompson C.J. Organization and topography of sensory responses in the internal capsule and nucleus Ventralis caudalis found during stereotactic surgery. Appl Neurophysiol 42:235-351, 1979.

13. Tasker R.R., Organ W., Hawrylyshyn P. The Thalamus and Midbrain of Man. Charles C. Thomas Publisher, Springfield, IL. 1982. 